\title{
Quantitative Measurement of White Matter Lesions Using Semi-automated Method
}

\author{
Yao Shieh ${ }^{1,2,3 *}$, Chien-Hung Chang1,2, Scott Goodwin ${ }^{3}$ \\ ${ }^{1}$ Chang Gung University, 259 Wenhua 1st Road, Guishan Dist. Taoyuan City, Taiwan. \\ ${ }^{2}$ Chang Gung Memorial Hospital, No.5, Fuxing St., Guishan Dist., Taoyuan City 333, Taiwan. \\ 3 University of California, Irvine 101 The City Drive South Orange, CA 92868 USA. \\ * Corresponding author. Tel.: 886+32118800 X 5378; email: yshieh@gmail.com \\ Manuscript submitted May 25, 2017; accepted August 10, 2017. \\ doi: 10.17706/ijbbb.2017.7.4.238-244
}

\begin{abstract}
The purpose of this study is to develop a semi-automated method to measure white matter lesion (WML) volumes in four different categories, and to assess the accuracy of the method. The four-category classification of white matter lesions has the potential of providing better etiological and functional relevance than the conventional two-category classification of periventricular and deep WMLs. The methodological steps are as follows. First, the Statistical Parametric Mapping (SPM) software is used to strip off the skull and scalp, and segment the FLAIR MR images of the brain into gray matter (GM), white matter (WM), and cerebrospinal fluid (CSF). Second, such segmentation is corrected manually. Third, the white matter is partitioned into four categories. Fourth, the mean, variance of each category is computed. Then the user selects a suitable threshold that differentiates WML from normal tissues. Finally, the WML volumes for each category are calculated. Three cases which represent mild, moderate and severe WML are used to test our proposed method. The overlap percentage between our method and the gold standard is $77 \%$, which compares favorably with the best results of $62.2 \%$ published in the literature. Based on the test results, our semi-automated method is promising to be a useful tool in quantitative measurement of white matter lesions.
\end{abstract}

Key words: Computer-aided decision making, medical imaging, quantitative measurement, segmentation, white matter lesion.

\section{Introduction}

White matter lesions (WML) have commonly been seen on MR images of elderly people and have been suggested to be associated with falls secondary to degradation in balance, gait, and mobility [1]-[3]. They have also been seen in patients who suffer from cognitive dysfunction, dementia, Alzheimer's disease, and multiple sclerosis [4]-[6].

The relationship between the location and volume of white matter lesions and the severity of disease has drawn attention from the research community. The MRI image sequence best used for quantitative volume measurement is the fluid attenuated inversion recovery (FLAIR) sequence [7], [8] because it manifests WMLs as brighter signals than normal tissues so that WMLs can be differentiated and measured more easily than on other sequences.

Over the years, researchers have attempted to develop fully automated methods to analyze hyperintensity white matter lesions (WML) from FLAIR images because automated methods are more 
reproducible whereas as compared to manual methods.. However, fully automated methods have not yet fully achieved the desired level of accuracy and robustness [9]-[12].

In addition, the conventional partition of WMLs into periventricular WMLs and deep WMLs [13] has been criticized because the distinction between them is ill defined. More recently, [14] suggested a four-category sub-classification, which takes into consideration the etiology, histopathology, functional correlates and imaging methodologies of WMLs. Specifically, this four-category sub-classification stratifies WMLs into juxtaventricular (within 3mm from ventricular surface), periventricular (3-13mm from ventricular surface), deep (between periventricular white matter and juxtacortical white matter), and juxtacortical (within $4 \mathrm{~mm}$ from corticomedullary junction) locations.

In this study, we propose a semi-automated method to partition the white matter into the four categories described above and to detect WML volumes within each of the four categories. Three cases which represent mild, moderate and severe WML are presented. The results show that our semi-automated method is sufficiently accurate to quantitatively measure WMLs.

\section{Methods}

Our proposed semi-automated method consists of five stages. First, the skull and the scalp are stripped off by SPM software. Second, the brain image is segmented into GM, WM, CSF and ventricles. Third, the white matter region is further classified into four categories: juxtaventricular, periventricular, deep, and juxtacortical categories. Fourth, the mean and variance for each of the four categories are computed, and in turn, the corresponding differentiating threshold for detecting white matter lesions (WML) is determined. Finally, WMLs in each of the four categories are detected and displayed.

\subsection{Subjects and MR Images}

We randomly selected 3 MRI scans of Chang Gung Memorial Hospital's patients: one having mild WML, another having moderate WML, and the third one having severe WML. MR images were acquired on the Gyroscan intera scanner manufactured by Philips Medical System. The study was approved by the Institutional Review Board (IRB) of Chang Gung Memorial Hospital.

Fluid attenuated inversion recovery (FLAIR) sequence MR Images were used in this study. FLAIR sequence highlights white matter hyperintensity lesions better than other MR sequences because it suppresses the unwanted high-intensity "noise" originating from cerebrospinal fluid.

\subsection{Brain Image Segmentation}

SPM open source software was used to segment the FLAIR image set into GM, WM and CSF, followed by human corrections because SPM is designed to segment brain images of normal subjects.

\subsection{Four-Category Sub-classification}

Once the FLAIR brain images were segmented into GM, WM and CSF, we focused on the WM region and further partitioned it into four categories, as proposed by Kim et al. [14]. These four categories are: juxtaventricular, periventricular, deep, and juxtacortical white matter regions, respectively.

Both juxtaventricular and periventricular WM regions are defined with respect to the surface of ventricles. The ventricles on FLAIR images have much lower intensity than the rest of the images, and therefore can be extracted reliably by thresholding technique followed by noise removing operations.

The juxtacortical region is defined as the image region within $4 \mathrm{~mm}$ from corticomedullary junction. In general, the SPM segmentation result is not precise enough to enable accurate delineation of the GM-WM boundary. This is where the user's manual correction is helpful. The original FLAIR image and its corresponding CSF map generated by the SPM are displayed side by side on the screen. The user uses a 
drawing pen or a mouse to delineate small regions to be added to or removed from the CSF region. The CSF region is updated instantly after each user's correction. Once finalized, the CSF boundary is determined and subsequently dilated by a suitable amount to lock onto the GM-WM boundary from which the juxtacortical region can be determined by computing the distance between each voxel and this GM-WM boundary. Finally, the deep WM region is determined by subtracting the juxtaventricular, periventricular, and juxtacortical regions.

\subsection{Determination of White Matter Lesion}

A voxel is classified to be a lesion voxel if its intensity is larger than the predetermined threshold that differentiates WML from normal tissues. To determine the differentiating threshold for a particular category, $\mathrm{TH}_{-}$category_i, the user draws a free-hand ROI that contains normal tissue only and no WMLs. Voxels of the same category within the ROI will be grouped together to compute that category's mean and variance. Subsequently, the differentiating threshold for each category is determined by the following equation:

$$
\text { TH_category_i }=\text { MEAN_ category_i }+\mathrm{k} * \text { STD_ category_i }
$$

where category_ $\mathrm{i} \in\{$ juxtaventricular, periventricular, deep, juxtacortical $\}$ and $\mathrm{k}$ is a user selectable variable with a default value being 2.5 .

Furthermore, the ROI is drawn on a user selected slice only once and the computed threshold is applied to all slices of the image set. Any pixel whose intensity is larger than its own relevant TH_category_i is classified to be a lesion pixel.

\subsection{Performance Evaluation}

The true WML volume is determined by a senior neurologist. The accuracy of the semi-automated WML method proposed herein is calculated using the concept of overlap percentage defined below:

$$
\text { Overlap }=\frac{\text { Computed WML volume } \cap \text { True WML volume }}{\text { Computed WML volume UTrue WML volume }}
$$

\section{Results}

Fig. 1 shows the processed results for slice \#13 of the mild WML case. The top left panel displays the original image. The forward and backward buttons allow the user to scroll through the whole set of images. The top right panel shows the segmentation result generated by the SPM software, which has stripped away the skull and scalp and separated the GM + WM areas (in white) from the CSF (in black). The ventricles are not shown in the figures intentionally to simplify the illustration. The top central panel shows the final results overlaid on the original image. The white matter has been classified into four categories: juxtaventricular (cyan), periventricular (green), deep (orange), and juxtacortical (blue) white matter regions, with white matter lesions shown in red.

Fig. 2 shows the processed results for slice \#12 of the moderate case. Note that the right middle portion of the image has brain atrophy resulting from earlier stroke. The SPM software cannot segment this portion correctly, and therefore, needs human intervention to make corrections. Fig. 3 shows the processed results for slice \#12 of the severe case.

The overall overlap percentage for the three test cases is $77 \%$ if a single value of parameter $\mathrm{k}$ in (1) is applied to the whole image set. However, the overall overlap percentage is $85 \%$ if a different value of $\mathrm{k}$ can be selected on a slice by slice basis. 

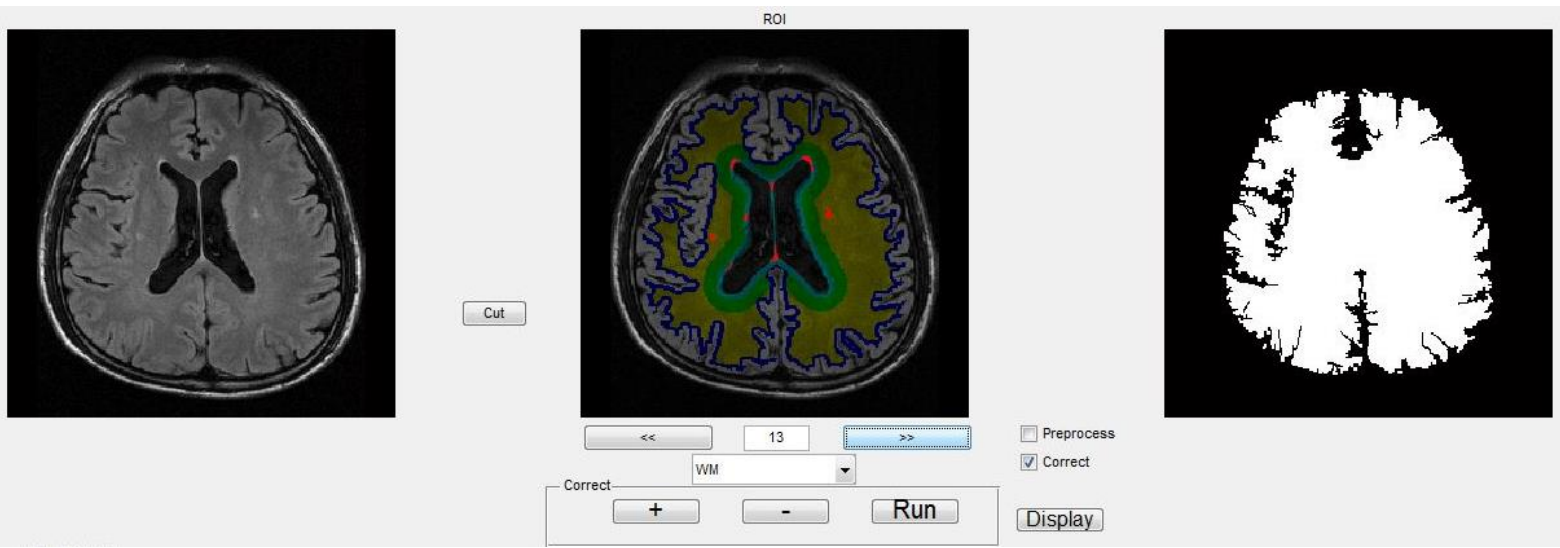

Fig. 1. The processed results of the mild WML case (slice \#13).
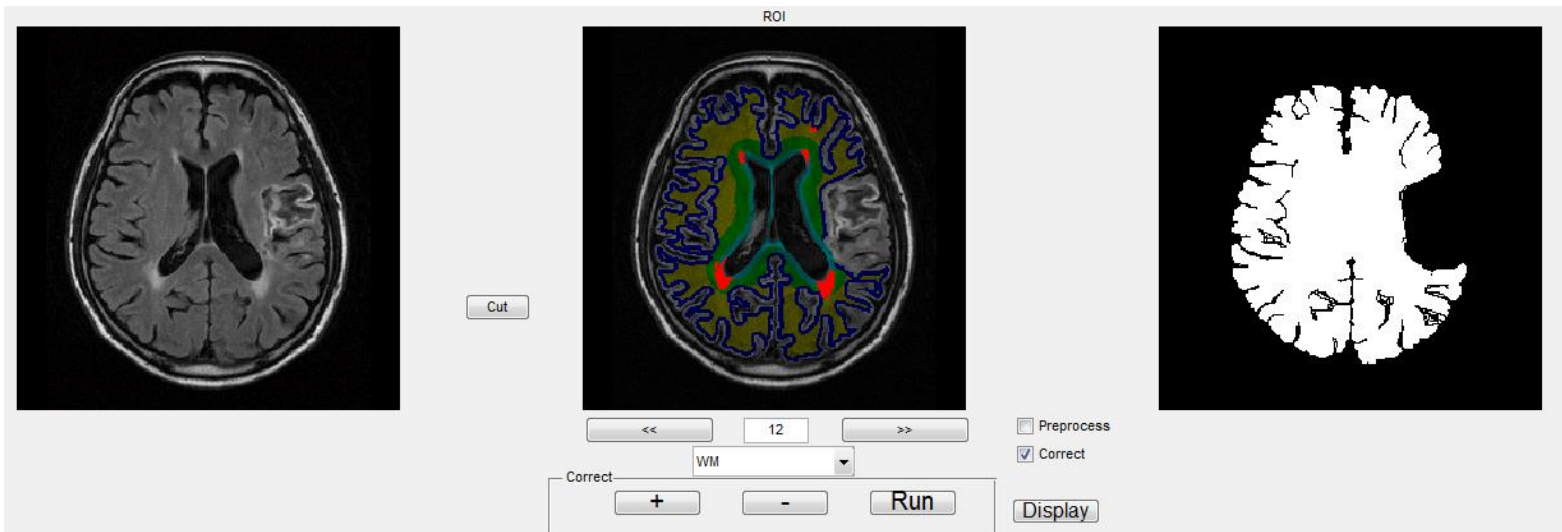

Fig. 2. The processed results of the moderate WML case (slice \#12).

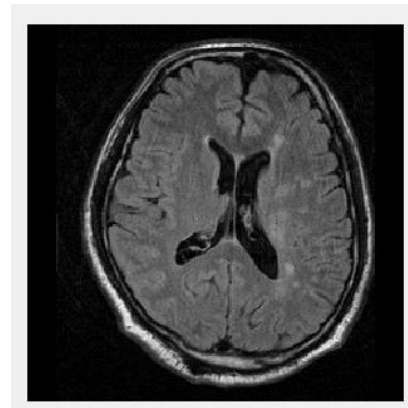

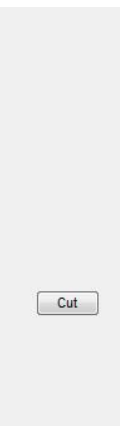

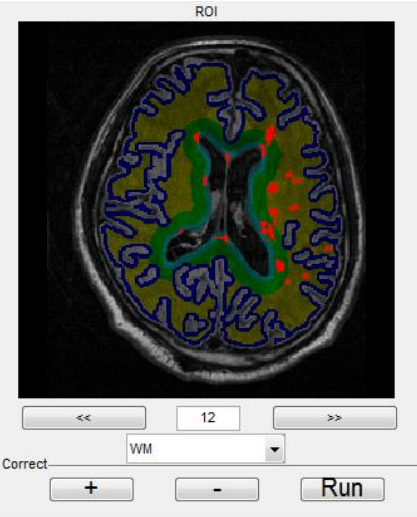

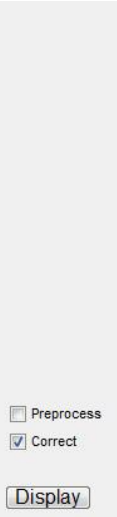

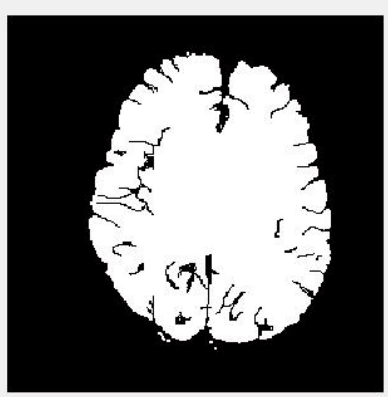

Display

Fig. 3. The processed results of the severe WML case (slice \# 12).

\section{Discussion}

Our method proposes a reasonable computer assisted diagnosis paradigm as applied to the challenge of quantitative measurement of WML volumes. Our method is based on SPM12, the latest version of the "standard" open source software for general purpose neuroimaging analysis, and therefore, builds upon the strength of the most powerful software commonly used in the neuroimaging research community. The SPM is designed for analyzing normal subjects. Its segmentation results on images containing white matter lesions may have errors, and so manual corrections are necessary.

Smart and colleagues [11] compared the performance of four fully automated methods that dealt with 
MR images acquired from real patients instead of from phantoms and reported the highest achievable overlap percentage to be $62.2 \%$. Our semi-automated method's overlap percentage of $77 \%$ and $85 \%$ compare favorably with the above published values. The preliminary results based on three test cases representing three levels of WML severity showed that our proposed method is promising. We are currently in the process of acquiring and testing a larger set of cases to further validate the robustness and suitability of our method in quantitative measurement of white matter lesions.

In the future, we will investigate the relationship between various types of brain functional impairment and white matter volumes of four categories to advance medical knowledge in this regard.

\section{Acknowledgment}

This research was supported by Ministry of Science and Technology, Taiwan, grant MOST104-2221-E-182-022, and Chang Gung Memorial Hospital Research Grant BMRPC12. The first author thanks Mr. C.C. Chen for his contributions in this study's computer programming.

\section{References}

[1] Leeuw, F., Groot, J. C., Achten, E., Oudkerk, M., Ramos, L., Heijboer, R., Hofman, A., Jolles, J., Gijn, J. V., \& Breteler, M. (2001). Prevalence of cerebral white matter lesions in elderly people: A population based magnetic resonance imaging study. The Rotterdam scan study. J Neurol Neurosurg Psychiatry, 70(1), 9-14.

[2] Wen, W., \& Sachdev, P. (2004). The topography of white matter hyperintensities on brain MRI in healthy 60- to 64-year-old individuals. NeuroImage, 22, 144-154.

[3] Ylikoski, A., Erkinjuntti, T., Raininko, R., Sarna, S., Sulkava, R., \& Tilvis, R. (1995). White matter hyperintensities on MRI in the neurologically nondiseased elderly. Analysis of cohorts of consecutive subjects aged 55 to 85 years living at home. Stroke, 26, 1171-1177.

[4] Bowen, B. C., Barker, W. W., Loewenstein, D. A., Sheldon, J., \& Duara, R. (1990). MR signal abnormalities in memory disorder and dementia. Am J Neuroradiol, 11, 283-290.

[5] Groot, J. C., Leeuw, F. E., Oudkerk, M., Gijn, J., Hofman, A., Jolles, J., \& Breteler, M. M. (2000). Cerebral white matter lesions and cognitive function: the Rotterdam Scan Study. Ann Neurology, 47, 145-151.

[6] Fazekas, F., Chawluk, J. B., Alavi, A., Hurtig, H. I., \& Zimmerman, R. A. (1987). MR signal abnormalities at $1.5 \mathrm{~T}$ in Alzheimer's dementia and normal aging. American Journal of Roentgenology, 149, 351-356.

[7] Coene, B. D., Hajnal, J. V., Gatehouse, P., Longmore, D. B., White, S. J., Oatridge, A., Pennock, J. M., Young, I. R., \& Bydder, G. M. (1992). MR of the brain using fluid-attenuated inversion recovery (FLAIR) pulse sequences. American Journal of Neuroradiology, 13, 1555-1564.

[8] Zawadzki, M. B., Atkinson, D., Detrick, M., Bradley W. G., \& Scidmore, G. (1996). Fluid-attenuated inversion recovery (FLAIR) for assessment of cerebral infarction. Initial clinical experience in 50 patients. Stroke, 27(7), 1187-1191.

[9] Jack, C. R., O’Brien, P. C., Rettman, D. W., Shiung, M. M., Xu, Y., Muthupillai, R., Manduca, A., Avula, R., \& Erickson, B. J. (2001). FLAIR histogram segmentation for measurement of leukoaraiosis volume. J Magn Reson Imaging, 14(6), 668-676.

[10] Lao, Z., Shena, D., Liu, D., Jawada, A. F., Melhem, E. R., Launer, L. J., Bryan, R. N., \& Davatzikos, C. (2008). Computer-assisted segmentation of white matter lesions in 3D MR images, using support vector machine. Academic Radiology, 15(3), 300-313.

[11] Smart, S. D., Firbank, M. J., \& O'Brien, J. T. (2011). Validation of automated white matter hyperintensity segmentation. Journal of Aging Research.

[12] Wu, M., Rosano, C., Butters, M., Whyte, E., Nable, M., Crooks, R., Meltzer, C. C., Reynolds, C. F., \& 
Aizenstein, H. J. (2006). A fully automated method for quantifying and localizing white matter hyperintensities on MR images. Psychiatry Research. 148, 133-142.

[13] DeCarli, C., Fletcher, E., Ramey, V., Harvey, D., \& Jagust, W. J. (2005). Anatomical mapping of white matter hyperintensities (WMH): Exploring the relationships between periventricular WMH, deep WMH, and total WMH burden. Stroke. 36, 50-55.

[14] Kim, K. W., MacFall, J. R., \& Payne, M. E. (2008). Classification of white matter lesions on magnetic resonance imaging in elderly persons. Biological Psychiatry, 64(4), 273-280.

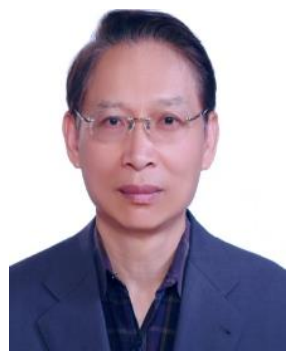

Yao Shieh received his Ph.D. degree in electrical engineering from Purdue University, Indiana, USA, in 1980. His research interests include pattern recognition, medical image analysis, medical informatics, machine learning, computer-aided diagnosis, and decision support systems.

He worked in the defense and commercial industry for 15 years before joining the academic community. He has been a Professor of the School of Medicine, University of California, Irvine since 2002, and assumed the role of Director of Medical Imaging \& Informatics in 2006. In 2009, he was invited to be a Visiting Professor of the Department of Electrical Engineering, Chang Gung University, and later became a regular Professor of CGU till now, while he still maintains the affiliation with UCI.

Professor Shieh is a member of IEEE, and AAPM (American Association of Physicists in Medicine). He was awarded the Certificate of Merit for the Picture Archiving \& Communication System (PACS) that he developed and demonstrated in the 1998 RSNA meeting at Chicago. He served as the General Chair for the $11^{\text {th }}$ IEEE Symposium on Computer-based Medical Systems in 1998. He chaired the Panel discussion on "Consumer Electronics @ Healthcare", at the IEEE ICCE Conference, Berlin, Germany in 2013, and chaired the invited session of Health/Medical Applications at IEEE ICCE Conference at Las Vegas, USA in 2014.. He served as the General Co-Chair of the IEEE-IMWS-bio 2015 Workshop. He has authored and co-authored over 50 peer-reviewed Journal and conference papers, and chapters for textbooks on Medical Informatics. He is also the Senior Editor of International Journal of Electronic Healthcare (IJEH, ISSN Print: 1741-8453, ISSN Online: 1741-8461) since 2006.

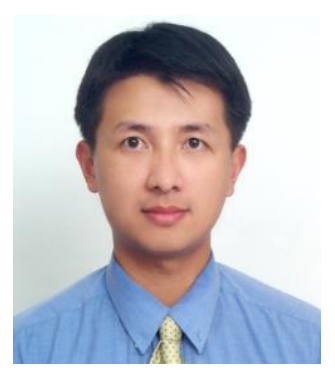

Chien-Hung Chang received his MD degree from China Medical College, Taichung, Taiwan, and his M.S. degree (medical informatics) from Chang Gung University, Tao-Yuan, Taiwan. He is currently a Ph.D. candidate at CGU.

He is the Chief of stroke division and stroke center, Department of Neurology, at Chang Gung Memorial Hospital (CGMH).

He has engaged in various research projects at CGMH for a period of six years, and has extensive experience in electronic medical stroke registry system of CGMH, medical image processing of stroke, and neuro-intervention such as carotid stent, intracranial stenting or acute stroke thrombectomy etc. He is well versed in systems that are pivotal to medical informatics, medical image visualization and neuro-intervention. Dr. Chang has written and published a number of scientific articles/papers in international journals and conference proceedings, and contributed chapters to books edited by others. 


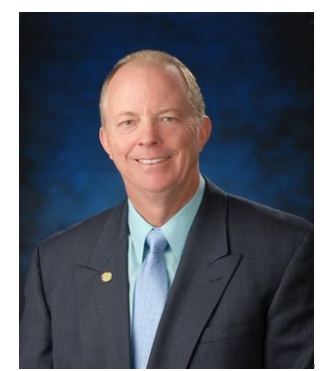

Scott Craig Goodwin, MD, FSIR was born July 15th, 1957 in Gardena, California. He received a Bachelor of Science in psychobiology Magna Cum Laude with departmental honors from University of California Los Angeles in 1979. He took his medical training at Harvard in Boston, Massachusetts, finishing in 1980. In 2017 he completed an MBA at George Washington University in Washington DC, finishing first in his class. Following medical school, he trained in Diagnostic Radiology and then Interventional Radiology at UCLA. He completed this training in 1989.

He was the Chief of Interventional Radiology at UCLA from 1994 to 2001. In 2001 he became the Chairman of Radiology at Wayne State University in Detroit Michigan where he was also the Chief of Imaging Services at Detroit Medical Center and the Chairman of the Board of Reynolds Radiology. Shortly thereafter he moved back to Los Angeles, California where he was the Vice Chairman of Radiology at UCLA and the Chief of Imaging Services at the Greater Los Angeles Veterans Administration Healthcare System. In 2007, he moved to the University of California Irvine (UCI) where he assumed the role of Hasso Brothers Professor and Chair of Radiological Sciences for the School of Medicine and Chief of Imaging Services for the Medical Center. In 2017 he assumed the role of President and CEO of Banner University Medical Group. In research he is best known for introducing uterine artery embolization for the treatment of uterine fibroids (UAE) in North America and he has authored or co-authored several of the landmark papers in the field including first reports on midterm results, effect of ovarian supply, sexual dysfunction, CT findings, pregnancy, unilateral UAE, and UAE versus myomectomy. Other notable UAE publications include the national research agenda, national training standards, reporting standards, registry results, and a NEJM review article. Whereas no researcher has been able to get federal funding for UAE research Dr. Goodwin and his colleagues were able to secure industry funding to perform several pivotal UAE trials. He has published over 80 peer review papers in addition to a book, various chapters, audio video works, abstracts, and posters.

Dr. Goodwin has been awarded fellowship status in the Society of Interventional Radiology and formerly served as President of the Society. Dr. Goodwin also previously served as Assistant Treasurer, Treasurer, Secretary, and President- elect of the Society. Prior Society activities include Medicare Carrier Advisory Committee Representative; Uterine Artery Embolization Task Force Chair and Society of Interventional Radiology Foundation Board Member. Dr. Goodwin has served on several Society committees that include: Government Affairs \&Health Care Policy; Technology Assessment; Program Directors; and Scientific Program Review. He is or has been a member of many other societies as well. He has been listed for many years in various Who's Who, Top Doctors, and Best Doctors lists. Dr. Goodwin has been active in the educational arena. He recently received an award from the UCI School of Medicine for his role in improving Radiological Sciences medical student education. The American Board of Radiology awarded him a lifetime service award for his contributions to the Diagnostic Radiology and Interventional Radiology board certification processes. As an officer of SIR, he played an important role in the creation of the primary certificate in Interventional Radiology. Upon his departure from UCI, the residents awarded him a lifetime achievement award. 